\title{
The effect of different volumes of high-intensity interval training on proinsulin in participants with the metabolic syndrome: a randomised trial
}

\author{
Joyce S. Ramos ${ }^{1}$ - Lance C. Dalleck ${ }^{2}$ - Fabio Borrani ${ }^{3,4}$ - Alistair R. Mallard ${ }^{1}$. \\ Bronwyn Clark $^{5} \cdot$ Shelley E. Keating ${ }^{1} \cdot$ Robert G. Fassett $^{1} \cdot$ Jeff S. Coombes $^{1}$
}

Received: 15 February 2016 / Accepted: 28 June 2016 / Published online: 1 August 2016

(C) Springer-Verlag Berlin Heidelberg 2016

\begin{abstract}
Aims/hypothesis The continuous demand for insulin in the face of insulin resistance, coupled with the glucolipotoxic environment associated with the metabolic syndrome (MetS), adversely affects the quality of insulin produced and secreted by the pancreatic beta cells. This is depicted by increased circulating intact proinsulin concentration, which is associated with increased MetS severity and risk of cardiovascular (CV) mortality. Highintensity interval training (HIIT) has been shown to reduce insulin resistance and other $\mathrm{CV}$ disease risk factors to a greater degree than moderate-intensity continuous training (MICT). We therefore aimed to investigate the impact of MICT and different volumes of HIIT on circulating intact proinsulin concentration. Methods This was a substudy of the 'Exercise in prevention of Metabolic Syndrome' (EX-MET) multicentre trial. Sixtysix individuals with MetS were randomised to 16 weeks of: (1) $\operatorname{MICT}(n=21,30 \mathrm{~min}$ at $60-70 \%$ peak heart rate
\end{abstract}

Electronic supplementary material The online version of this article (doi:10.1007/s00125-016-4064-7) contains peer-reviewed but unedited supplementary material, which is available to authorised users.

Jeff S. Coombes

jcoombes@uq.edu.au

1 Centre for Research on Exercise, Physical Activity and Health, School of Human Movement and Nutrition Sciences, The University of Queensland, Blair Drive, St Lucia, QLD, Australia

2 Recreation, Exercise, and Sport Science Department, Western State Colorado University, Gunnison, CO, USA

3 Institut des Sciences du Sport de l'Université de Lausanne (ISSUL), University of Lausanne, Lausanne, Switzerland

4 Department of Physiology, Faculty of Biology and Medicine, Lausanne University, Lausanne, Switzerland

5 School of Public Health, Faculty of Medicine and Biomedical Sciences, The University of Queensland, Brisbane, QLD, Australia
[HRpeak], five times/week); (2) 4HIIT ( $n=22,4 \times 4 \mathrm{~min}$ bouts at $85-95 \%$ HRpeak, interspersed with 3 min of active recovery at 50-70\% HRpeak, three times/week); or (3) 1 HIIT $(n=23,1 \times 4$ min bout at $85-95 \%$ HRpeak, three times/week). A subanalysis investigated the differential impact of these training programmes on intact proinsulin concentration in MetS individuals with type 2 diabetes (MICT, $n=6$; 4HIIT, $n=9$; 1 HIIT, $n=12$ ) and without type 2 diabetes (MICT, $n=15$; 4HIIT, $n=13$; $1 \mathrm{HIIT}, n=11)$. Intact proinsulin, insulin and C-peptide concentrations were measured in duplicate via ELISA, following a $12 \mathrm{~h}$ fast, before and after the exercise programme. Fasting intact proinsulin concentration was also expressed relative to insulin and C-peptide concentrations. Results Following the exercise training, there were no significant $(p>0.05)$ changes in fasting intact proinsulin concentration indices in all participants (pre- vs post-programme proinsulin, proinsulin:insulin, proinsulin:C-peptide: MICT 19\% decrease, $6 \%$ increase, $4 \%$ increase; 4 HIIT $19 \%$ decrease, $8 \%$ decrease, $11 \%$ decrease; 1 HIIT $34 \%$ increase, $49 \%$ increase, $36 \%$ increase). In participants who did not have type 2 diabetes, only 4HIIT significantly $(p<0.05)$ reduced fasting intact proinsulin concentration indices from pre to post intervention (pre- vs post-programme proinsulin, proinsulin:insulin, proinsulin:C-peptide: 4HIIT 32\% decrease, $26 \%$ decrease, $32 \%$ decrease, $p<0.05$; 1HIIT, $14 \%$ increase, $32 \%$ increase, $16 \%$ increase, $p>0.05$; MICT $27 \%$ decrease, $17 \%$ decrease, $11 \%$ decrease), with a group $\times$ time interaction effect, indicating a greater reduction in intact proinsulin indices following 4HIIT compared with MICT and 1HIIT. There were no significant $(p>0.05)$ changes in intact proinsulin concentration indices in participants with type 2 diabetes.

Conclusions/interpretation Higher-volume HIIT (4HIIT) improved insulin quality in MetS participants without type 2 diabetes.

Trial registration: ClinicalTrials.gov NCT01676870 
Funding: The study was funded by the Norwegian University of Science and Technology and from an unrestricted research grant from the Coca-Cola company. Funding for the collection of physical activity data was derived from a 'UQ New Staff Start Up' grant awarded to B. Clark.

Keywords Glycaemic control · Insulin resistance $\cdot$ Interval training $\cdot$ Pancreatic beta cell function $\cdot$ Proinsulin

$\begin{array}{ll}\text { Abbreviations } \\ \text { BF\% } & \text { Body fat } \% \\ \text { CRF } & \text { Cardiorespiratory fitness } \\ \text { CV } & \text { Cardiovascular } \\ \text { CVD } & \text { Cardiovascular disease } \\ \text { DI } & \text { Disposition index } \\ \text { DBP } & \text { Diastolic BP } \\ \text { ER } & \text { Endoplasmic reticulum } \\ \text { EX-MET } & \text { Exercise in prevention of Metabolic } \\ & \text { Syndrome } \\ \text { FFM } & \text { Free fat mass } \\ \text { GSIS } & \text { Glucose-stimulated insulin secretion } \\ \text { HDL-C } & \text { HDL-cholesterol } \\ \text { HIIT } & \text { High-intensity interval training } \\ \text { HOMA-B } & \text { HOMA of beta cell function } \\ \text { HRpeak } & \text { Peak heart rate } \\ \text { IDF } & \text { International Diabetes Federation } \\ \text { IFG } & \text { Impaired fasting glucose } \\ \text { IGT } & \text { Impaired glucose tolerance } \\ \text { IR } & \text { Insulin resistance } \\ \text { MetS } & \text { Metabolic syndrome } \\ \text { MICT } & \text { Moderate-intensity continuous training } \\ \text { MVPA } & \text { Moderate to vigorous physical activity } \\ \text { PGC-1 } \alpha & \text { Peroxisome proliferator-activated } \\ & \text { receptor- } \gamma \text { coactivator 1, alpha } \\ \text { ROS } & \text { Reactive oxygen species } \\ \text { SBP } & \text { Systolic BP } \\ \text { TF\% } & \text { Trunk fat } \% \\ \dot{V} \text { O } \\ \text { Weak } & \text { Peak oxygen uptake } \\ \text { WC } & \text { Waist circumference }\end{array}$

\section{Introduction}

Insulin resistance (IR) has been proposed as the central mediating factor in the emergence of cardiovascular disease (CVD) risk factors, including hyperglycaemia, dyslipidaemia, hypertension and central obesity, which collectively constitute the metabolic syndrome (MetS) [1]. Thus, the presence of MetS inevitably imposes an increased burden on the pancreatic beta cells to hypersecrete insulin in an attempt to regulate blood glucose, leading to impaired pancreatic beta cell function [2].
The continuous demand for insulin in the face of IR, coupled with the glucolipotoxic environment associated with MetS [3], adversely affects the quality of insulin produced and secreted by the pancreatic beta cells $[4,5]$. Insulin synthesis is initiated in the endoplasmic reticulum (ER) of the pancreatic beta cells, where proinsulin is cleaved from its folded preprohormone state (preproinsulin). The proinsulin produced is then packaged by the trans-Golgi to form secretory granules that are then acted on by proteases (carboxypeptides), cleaving C-peptide from the proinsulin molecule to form mature insulin. However, increased insulin demand in the presence of hyperglycaemia leads to defective processing or premature release of proinsulin molecules $[4,5]$ that have lower affinity for tissue insulin receptors, showing only about $10-20 \%$ of the capacity of insulin to induce glucose uptake [6]. Therefore, increased intact proinsulin concentration has been identified as a key marker of poor insulin quality, and thus impaired beta cell function [7].

A recent study that followed 9396 non-type 2 diabetic individuals for 6 years showed fasting intact proinsulin concentration to be a predictor of both the worsening of hyperglycaemia and incidence of type 2 diabetes, with no difference in predictive capacity compared with intact proinsulin concentration level derived from a dynamic state [8]. Moreover, several studies have also found increased fasting intact proinsulin concentration to be an independent predictor of all-cause and cardiovascular (CV) mortality $[9,10]$.

Increased fasting intact proinsulin concentration has been linked with increased severity of MetS [11] and therefore investigations establishing the efficacy of therapies that can modulate intact proinsulin concentration levels in MetS individuals are warranted. Increased cardiorespiratory fitness (CRF) with exercise training has been established as an antidote for different MetS components [12, 13]. However, the effects of exercise on fasting intact proinsulin concentration have not yet been established. Although moderate-intensity continuous training (MICT) has long been established as an efficacious intervention to improve CRF [13] and to reduce or prevent the development of cardiometabolic disorders [14, 15], the time commitment recommended for benefit ( $\geq 30 \mathrm{~min} / \mathrm{session}, 5-7$ days/week) is a barrier for long-term exercise adherence [16]. Meta-analyses have revealed that a more time-efficient form of exercise known as high-intensity interval training (HIIT) improves CRF [17] and IR [18] to a greater extent than MICT. Moreover, Tjonna et al [19] have shown that a low-volume HIIT $(1 \mathrm{HIIT}, 1 \times 4 \mathrm{~min}$ interval at 90\% peak heart rate [HRpeak]) improves CRF to a similar extent as a high-volume HIIT (4HIIT, $4 \times 4$ min intervals at 90\% HRpeak, separated by 3 min active recovery). Thus, given that CRF has been shown to be inversely associated with IR and beta cell dysfunction in individuals across the entire glucose tolerance continuum [20], it could be inferred that the low-volume 1HIIT protocol is sufficient to improve 
insulin quality. However, the specific impact of any dose of HIIT relative to MICT on insulin quality or beta cell function in MetS individuals has yet to be investigated.

Therefore, the primary aim of the current study was to investigate the impact of different volumes of HIIT (4HIIT and 1HIIT) and MICT on fasting intact proinsulin concentration indices in MetS individuals. As secondary outcome measures, the impact of these different exercise doses on CRF, IR and other markers of beta cell function were also determined. A subanalysis was also performed to investigate the differential impact of these training programmes on fasting intact proinsulin concentration, IR and other beta cell function indices in MetS individuals with type 2 diabetes and without type 2 diabetes. We hypothesised that, in a dose-dependent manner, HIIT interventions (4HIIT > 1HIIT) would be more efficacious relative to MICT in improving all outcome measures.

\section{Methods}

Participants and study design Ninety-nine individuals diagnosed with MetS according to the International Diabetes Federation (IDF) criteria, were recruited (January 2013 to August 2015). This is a sub-study of the 'Exercise in prevention of Metabolic Syndrome' (EX-MET) multicentre trial (ClinicalTrials.gov NCT01676870). The present substudy reports data collected exclusively at the Brisbane site where participants were also requested to undertake an OGTT. The fasting intact proinsulin concentration and other pancreatic beta cell function indices, and parameters derived from the OGTT were the endpoints specific to the present study. All other outcome measures reported in the current study are background information/endpoints that are either part of the EX-MET trial (MetS risk factors, CRF and HOMA-IR), or have been published previously (body composition) [21]. Fig. 1 presents a consort diagram of participant flow throughout this substudy. Sample size was calculated using an anticipated mean difference in intact proinsulin concentration reduction of 0.62 (power $=0.80$, alpha $=0.05$ for 2-tailed test) between the HIIT and MICT groups. This was based on 1) a previous meta-analysis showing a similar mean difference in reduction of IR between HIIT and MICT in MetS or type 2 diabetes [18]; and 2) that the continuous demand for insulin in the face of IR negatively affects the quality of insulin processed by the pancreatic beta cells [5]. See electronic supplementary material (ESM) Methods for further details on the recruitment process, exclusion criteria and randomisation procedure.

Participants underwent several tests at the university's laboratory (Human Movement and Nutrition Sciences Building, The University of Queensland, St Lucia, QLD, Australia) before and after the 16 week interventions to measure the following: (1) MetS risk factors and body composition; (2) fasting intact proinsulin concentration indices; (3) CRF;
(4) insulin sensitivity; and (5) other beta cell function indices. See ESM Methods for further details.

MetS risk factors The following assessments were conducted at a $12 \mathrm{~h}$ fasted state to determine participants eligibility for the study: (1) fasting lipid profile and glucose level; (2) brachial systolic BP (SBP) and diastolic BP (DBP); and (3) anthropometric measures (weight, height, waist circumference [WC] and hip circumference $[\mathrm{HC}]$ ). In-depth details of these assessments have been reported previously [22]. After the 16 week training interventions, a tester blinded to the training group allocation performed the subsequent series of tests.

Proinsulin, insulin sensitivity and other beta cell function measures Serum and plasma samples were obtained from blood collected from the participants' antecubital vein following a $12 \mathrm{~h}$ overnight fast for later analysis of intact proinsulin concentration (human intact proinsulin ELISA, EZHIPI-17K, Merck Millipore, Darmstadt, Germany), fasting glucose concentration (Rx Daytona Plus, Randox Laboratories, Crumlin, County Antrim, UK), C-peptide and insulin concentrations (electrochemiluminescence immunoassay [ECLIA], Cobas e411 immunoassay analyzer, Roche Diagnostics, Indianapolis, IN, USA). $\mathrm{HbA}_{1 \mathrm{c}}$ was also measured from a whole-blood sample (Rx Daytona Plus). Ratios of fasting intact proinsulin concentration to insulin or C-peptide concentration (proinsulin:insulin and proinsulin:C-peptide) were calculated. See ESM Methods for further details.

HOMA-IR and HOMA of beta cell function (HOMA-B) were used to determine IR and insulin secretion at a steady state, calculated via the HOMA2 calculator version 2.2 [23]. The basal disposition index (basal DI = HOMA-B/HOMA-IR) was then calculated to determine beta cell function at a steady state condition, given that the amount of insulin secreted is highly dependent upon the prevailing IR magnitude. A $2 \mathrm{~h}$ OGTT was also performed in a subgroup of participants $(n=32)$ to determine IR and beta cell function in a dynamic condition. See ESM Methods for further details.

Cardiorespiratory fitness CRF depicted as the peak oxygen uptake $\left(\dot{V} \mathrm{O}_{2 \text { peak }}\right)$ was measured via indirect calorimetry using the Metamax II system (Cortex, Leipzig, Germany) or Parvo Medics TrueOne 2400 system (Parvomedics, Sandy, UT, USA) during a graded maximal exercise test. See ESM Methods for further details.

Body composition Body composition was assessed at baseline and after the 16 week training programme via dual-energy x-ray absorptiometry (Hologic Discovery W Apex software version 3.3; Hologic, Bedford, MA, USA). A 3 day food diary was also administered before and after the training programme 


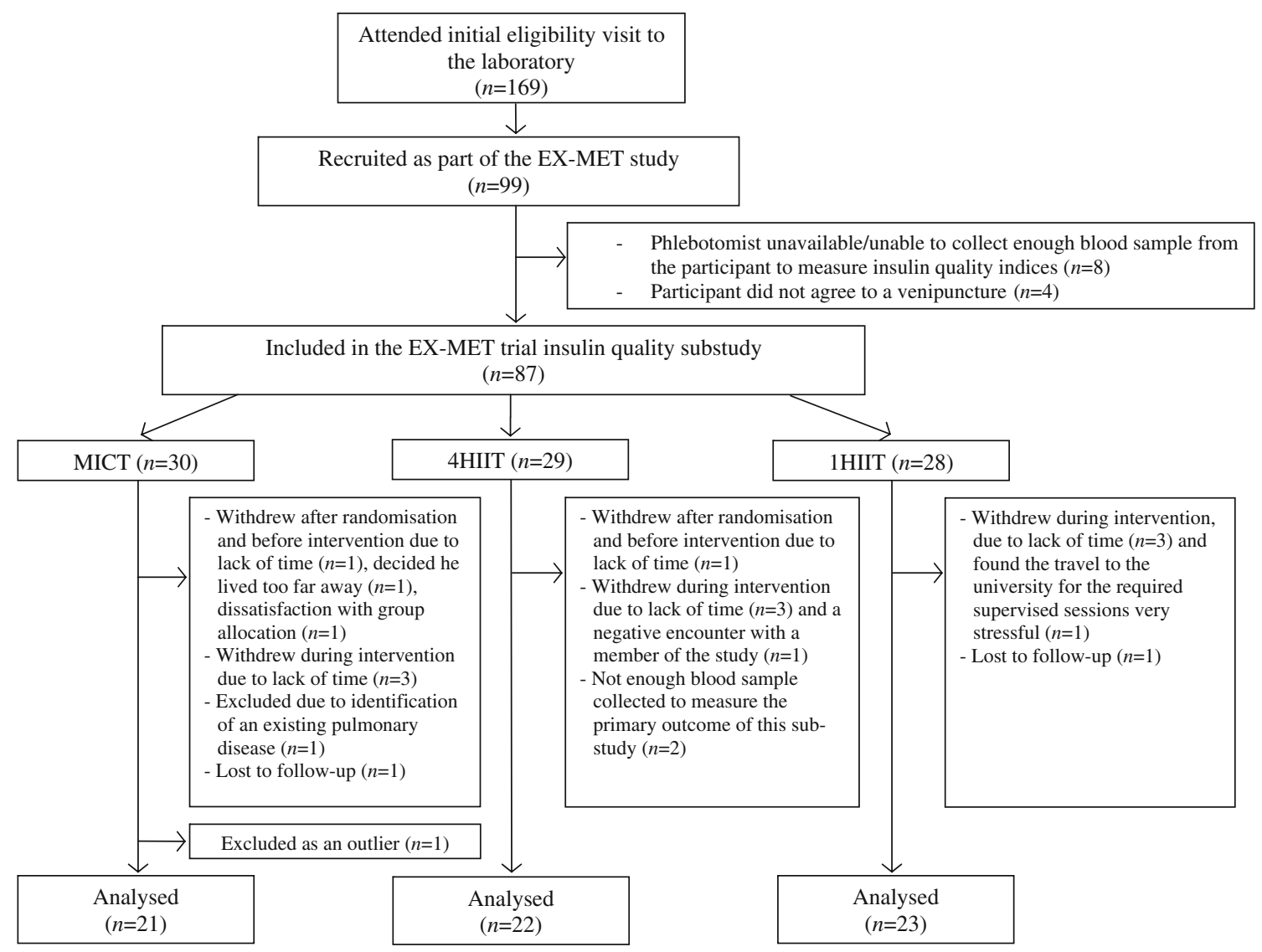

Fig. 1 Flow chart for insulin quality substudy

to determine total energy intake. See ESM Methods for further details.

Training protocol The HIIT groups trained three times per week, whilst the MICT group trained five times per week. Each MICT was $30 \mathrm{~min}$ in duration at a continuous pace and target intensity of 60-70\% HRpeak or rate of perceived exertion (RPE) of 11-13 on the Borg scale (Fig. 2a). The 4HIIT group trained for 38 min per session (Fig. 2b), whilst each session of the 1HIIT protocol was approximately half of this duration (17 min) (Fig. 2c). The metabolic equivalent (MET) of the average workload throughout the intervention was calculated [24], and used to calculate energy expended per min based on the following assumptions: $1 \mathrm{MET}=3.5 \mathrm{ml} \mathrm{kg}^{-1}$ $\min ^{-1}$ and 11 of $\mathrm{O}_{2}=20.92 \mathrm{~kJ}$ (5 kcal). See ESM Methods for further details. Moderate to vigorous physical activity (MVPA) time ( $\mathrm{min} /$ day) was also measured before and after the exercise programme using the activPAL device (Version 3 , $\mathrm{Pal}$ Technologies, Glasgow, UK) as described previously $[25,26]$.

Statistical analysis The SPSS version 22 software package (IBM, New York, NY, USA) was used to analyse all data. One-way ANOVA or Kruskal-Wallis, and $\chi^{2}$ tests were used to compare baseline values, as well as training adherence, between intervention groups. Within-group differences in continuous variables were analysed via a paired $t$ test or Wilcoxon test. Significant within-group changes in intact proinsulin concentration indices over time were further assessed via mixed linear model regression analysis.

Group $\times$ time interaction effects were examined via ANCOVA. $\eta^{2}$ group $\times$ time interaction effect sizes were calculated as the between-group sum of squares divided by the total sum of squares. McNemar's test was used to determine whether there was a significant difference between the number of participants diagnosed with MetS according to the IDF criteria from pre to post intervention. Significance level was set at $p<0.05$. See ESM Methods for further details.

\section{Results}

Sixty-six participants completed the exit assessment for the primary outcome of the study (Fig. 1). There were no significant group differences in demographics and medication intake at baseline (Table 1). All baseline values of physiological variables assessed were also similar across all groups (Tables 2, 3, 4 and 5), except for baseline total glucose AUC, where there was a significant difference between MICT and 1HIIT groups $(p<0.01)$. The MICT, 4HIIT and 
Fig. 2 Schematic representation of MICT (a), 4HIIT (b) and $1 \mathrm{HIIT}(\mathbf{c})$

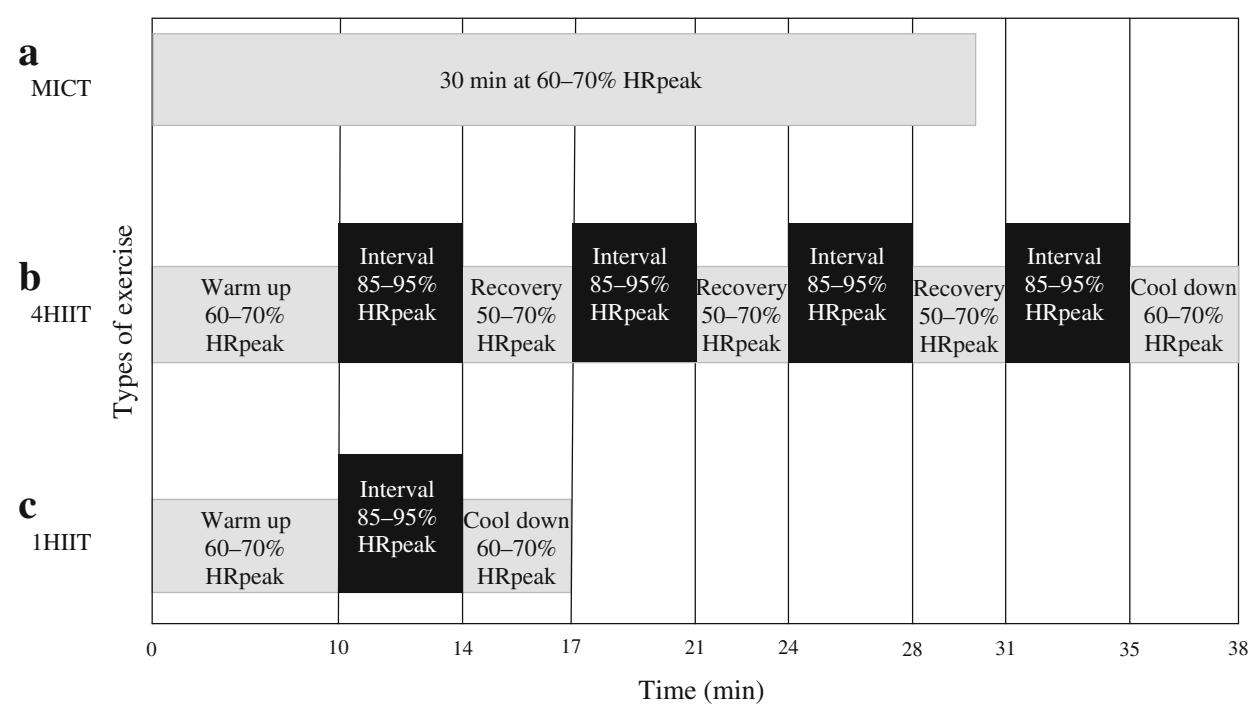

1HIIT groups completed $87 \pm 14 \%, 88 \pm 9 \%$ and $88 \pm 16 \%$ (group difference, $p=0.73$ ) of the training prescribed, respectively, with an estimated total of $59,199 \pm 17,209 \mathrm{~kJ}(14,149$ $\pm 4113 \mathrm{kcal}), 65,534 \pm 23,493 \mathrm{~kJ}(15,663 \pm 5615 \mathrm{kcal})$ and $24,920 \pm 9381 \mathrm{~kJ}(5956 \pm 2242 \mathrm{kcal})$ expended over the 16 week programme, respectively (group difference, $p<0.001)$. This equated to $\sim 3699 \pm 1075 \mathrm{~kJ} /$ week $(884$ $\pm 257 \mathrm{kcal} /$ week $), 4096 \pm 1469 \mathrm{~kJ} /$ week $(979 \pm 351 \mathrm{kcal} /$ week $)$ and $1556 \pm 586 \mathrm{~kJ} /$ week $(372 \pm 140 \mathrm{kcal} /$ week $)$, respectively (group difference, $p<0.001$ ). There were significant differences in estimated $\mathrm{kcal} /$ week and total kcal between the 1 HIIT and both MICT and 4HIIT groups, but not between MICT and 4HIIT. The average exercise intensities for MICT, 4 HIIT and 1 HIIT were $7 \pm 3,20 \pm 7$ and $20 \pm 6 \mathrm{kcal} / \mathrm{min}$ (group difference, $p<0.01$ ), respectively, with a significant difference

Table 1 Participant characteristics

\begin{tabular}{lllll}
\hline Variable & $\begin{array}{l}\text { MICT } \\
(n=21)\end{array}$ & $\begin{array}{l}\text { 4HIIT } \\
(n=22)\end{array}$ & $\begin{array}{l}\text { 1HIIT } \\
(n=23)\end{array}$ & $p$ value \\
\hline Demographics & & & & \\
$\quad$ Age (years) & $57 \pm 9$ & $56 \pm 10$ & $58 \pm 7$ & 0.72 \\
$\quad$ Male sex (\%) & 71 & 55 & 61 & 0.52 \\
$\quad$ Type 2 diabetes (\%) & 29 & 41 & 52 & 0.28 \\
$\quad$ Hypertensive (\%) & 71 & 73 & 78 & 0.86 \\
Medications & & & & \\
$\quad$ Angiotensin II blocker (\%) & 43 & 45 & 52 & 0.81 \\
$\quad$ Calcium antagonist (\%) & 10 & 23 & 13 & 0.45 \\
$\quad$ Beta-blocker (\%) & 14 & 0 & 17 & 0.13 \\
$\quad$ Statin (\%) & 33 & 50 & 57 & 0.29 \\
$\quad$ Aspirin ${ }^{\text {a }}(\%)$ & 24 & 18 & 17 & 0.85 \\
Metformin (\%) & 24 & 27 & 35 & 0.71 \\
\hline
\end{tabular}

Data shown as mean $\pm \mathrm{SD}$ or $\%$

${ }^{\mathrm{a}}$ Acetylsalicylic acid between MICT and HIIT groups. The number of participants who trained on a cycle ergometer was similar between all training groups (MICT, $n=4$; 4HIIT, $n=3$; 1 HIIT, $n=5$; group difference, $p=0.77$ ). There were no reported physical injuries that were directly related to the prescribed interventions in participants included in this substudy.

Intact proinsulin concentration indices In all participants, we found no significant changes in fasting intact proinsulin concentration indices following the training interventions (Table 2). A subanalysis that compared participants with or without type 2 diabetes showed that 4HIIT significantly decreased the intact proinsulin concentration indices in non-type 2 diabetic individuals from pre to post intervention (proinsulin, $-32 \%$; proinsulin:insulin, $-26 \%$; proinsulin:C-peptide, $-32 \%$; see Table 3 ). We also detected a small to medium group $\times$ time interaction effect on all intact proinsulin indices, indicating a positive adaptation following 4HIIT compared with MICT and 1HIIT in non-type 2 diabetic individuals (Table 3). In contrast, there were no significant changes in fasting intact proinsulin concentration indices in those with type 2 diabetes following the training interventions (Table 4).

Insulin resistance, glycaemic control and other beta cell function indices derived from a fasting steady-state IR measured at a fasted steady-state condition (HOMA-IR) significantly decreased following MICT and 4HIIT by $-23 \%$ and $-14 \%$, respectively, but not following 1HIIT (group difference, $p>0.05)$. In our subanalysis, the same pattern in HOMA-IR improvement was evident in non-type 2 diabetic individuals (Table 3), with no significant change in type 2 diabetic participants (Table 4). Moreover, only the 4HIIT group had a significant decrease in insulin secretion (HOMA-B), $-11 \%$ (Table 2), following the intervention. Again, our subanalysis showed that this pattern was only 
Table 2 Changes in beta cell function and cardiometabolic factors following different types of training in all participants

\begin{tabular}{|c|c|c|c|c|c|c|c|c|c|c|c|c|c|c|}
\hline \multirow[t]{3}{*}{ Variable } & \multicolumn{4}{|c|}{$\operatorname{MICT}(n=21)$} & \multicolumn{4}{|c|}{$4 \mathrm{HIIT}(n=22)$} & \multicolumn{4}{|c|}{ 1HIIT $(n=23)$} & \multicolumn{2}{|c|}{ Between groups } \\
\hline & \multicolumn{2}{|l|}{ Pre } & \multicolumn{2}{|l|}{ Post } & \multicolumn{2}{|l|}{ Pre } & \multicolumn{2}{|l|}{ Post } & \multicolumn{2}{|l|}{ Pre } & \multicolumn{2}{|l|}{ Post } & \multirow[b]{2}{*}{$p$ value } & \multirow[b]{2}{*}{ Effect size } \\
\hline & Mean & $\mathrm{SD}$ & Mean & SD & Mean & SD & Mean & SD & Mean & SD & Mean & SD & & \\
\hline \multicolumn{15}{|l|}{ Beta cell function } \\
\hline Basal DI ${ }^{\mathrm{a}}$ & 69.9 & 34.2 & 82.3 & $32.9^{*}$ & 62.0 & 34.0 & 66.6 & 41.4 & 59.3 & 28.9 & 66.4 & 40.8 & 0.52 & 0.02 \\
\hline Intact proinsulin (pmol/l) & 3.1 & 3.7 & 2.5 & 2.9 & 4.2 & 3.8 & 3.4 & 3.9 & 4.4 & 4.4 & 5.9 & 7.5 & 0.30 & 0.03 \\
\hline Intact proinsulin:insulin $\left(\times 10^{-2}\right)$ & 3.5 & 4.9 & 3.7 & 5.5 & 3.7 & 3.0 & 3.4 & 3.3 & 4.1 & 3.7 & 6.1 & 7.1 & 0.52 & 0.02 \\
\hline Intact proinsulin:C-peptide $\left(\times 10^{-3}\right)$ & 2.7 & 3.4 & 2.8 & 3.5 & 3.5 & 3.0 & 3.1 & 3.1 & 3.6 & 3.0 & 4.9 & 5.2 & 0.39 & 0.03 \\
\hline \multicolumn{15}{|l|}{ Glucose and insulin metabolism } \\
\hline Fasting glucose (mmol/l) & 6.3 & 1.3 & 5.9 & 0.9 & 7.1 & 2.6 & 7.2 & 3.0 & 7.0 & 1.7 & 7.0 & 1.9 & 0.11 & 0.05 \\
\hline Fasting insulin (pmol/1) & 114 & 69 & 89 & $58^{*}$ & 106 & 38 & 91 & 39 & 107 & 56 & 99 & 64 & 0.41 & 0.03 \\
\hline C-peptide (nmol/l) & 1.20 & 0.37 & 0.97 & $0.30 *$ & 1.14 & 0.27 & 1.02 & $0.32 *$ & 1.12 & 0.29 & 1.07 & 0.39 & 0.12 & 0.07 \\
\hline $\mathrm{HbA}_{1 \mathrm{c}}(\%)$ & 5.9 & 0.7 & 5.7 & 0.5 & 6.8 & 2.2 & 6.8 & 2.4 & 6.6 & 1.3 & 6.4 & 0.8 & 0.52 & 0.03 \\
\hline $\mathrm{HbA}_{1 \mathrm{c}}(\mathrm{mmol} / \mathrm{mol})$ & 40.4 & 7.8 & 39.2 & 5.9 & 50.6 & 24.1 & 50.8 & 26.2 & 48.4 & 14.4 & 46.4 & 8.8 & 0.52 & 0.03 \\
\hline HOMA-IR (insulin) & 2.2 & 1.3 & 1.7 & $1.1^{*}$ & 2.1 & 0.9 & 1.8 & $0.8^{*}$ & 2.1 & 1.1 & 2.0 & 1.2 & 0.32 & 0.04 \\
\hline HOMA-B (insulin) & 105.8 & 48.5 & 98.6 & 48.8 & 93.0 & 40.7 & 83.0 & $34.9 *$ & 85.7 & 40.6 & 79.5 & 42.9 & 0.77 & 0.01 \\
\hline HOMA-B (C-peptide) & 126.0 & 36.3 & 117.2 & 34.2 & 113.8 & 41.2 & 103.9 & $39.0^{*}$ & 104.5 & 34.6 & 100.9 & 35.5 & 0.51 & 0.02 \\
\hline \multicolumn{15}{|l|}{$\mathrm{CRF}$} \\
\hline Absolute $\dot{V} \mathrm{O}_{2 \text { peak }}(1 / \mathrm{min})$ & 2.7 & 0.8 & 2.8 & 0.7 & 2.4 & 0.7 & 2.7 & $0.9^{*}$ & 2.3 & 0.7 & 2.5 & $0.8^{*}$ & 0.15 & 0.06 \\
\hline Relative $\dot{V} \mathrm{O}_{2 \text { peak }}\left(\mathrm{ml} \mathrm{kg}^{-1} \min ^{-1}\right)$ & 27.9 & 7.5 & 28.7 & 6.8 & 24.6 & 5.8 & 28.2 & $7.3 *$ & 25.8 & 6.5 & 27.9 & $6.7 *$ & 0.15 & 0.06 \\
\hline \multicolumn{15}{|c|}{ MetS risk factors and anthropometric measures } \\
\hline HDL-C (mmol/l) & 1.13 & 0.35 & 1.21 & 0.40 & 1.01 & 0.33 & 1.09 & 0.36 & 1.15 & 0.43 & 1.28 & $0.39 *$ & 0.57 & 0.02 \\
\hline Triacylglycerol (mmol/l) & 1.73 & 0.76 & 1.62 & 0.91 & 2.30 & 1.21 & 2.17 & 1.18 & 1.86 & 0.70 & 1.67 & 0.58 & 0.63 & 0.01 \\
\hline SBP (mmHg) & 132 & 13 & 125 & $11^{*}$ & 129 & 12 & 127 & 10 & 135 & 13 & 126 & $13^{*}$ & 0.19 & 0.04 \\
\hline DBP (mmHg) & 86 & 10 & 81 & $7 *$ & 84 & 7 & 80 & $7 *$ & 82 & 7 & 79 & 7 & 0.99 & $<0.001$ \\
\hline WC & 107 & 12 & 105 & $13^{*}$ & 105 & 11 & 104 & 10 & 103 & 13 & 100 & $12 *$ & 0.39 & 0.03 \\
\hline Waist:hip ratio & 0.94 & 0.09 & 0.92 & $0.08^{*}$ & 0.91 & 0.08 & 0.90 & 0.07 & 0.94 & 0.09 & 0.91 & $0.07 *$ & 0.59 & 0.01 \\
\hline Weight (kg) & 99 & 18 & 98 & 19 & 98 & 17 & 97 & 17 & 92 & 21 & 90 & 20 & 0.94 & $<0.001$ \\
\hline $\mathrm{BF} \%$ & 38.9 & 8.8 & 38.5 & 9.1 & 42.3 & 6.4 & 41.8 & 7.0 & 39.5 & 7.3 & 39.0 & 7.4 & 0.97 & $<0.001$ \\
\hline $\mathrm{TF} \%$ & 42.3 & 8.5 & 41.8 & 8.6 & 44.9 & 5.5 & 44.6 & 6.1 & 42.9 & 6.4 & 42.4 & 6.2 & 0.93 & $<0.001$ \\
\hline FFM (kg) & 56.9 & 11.8 & 56.5 & 12.1 & 54.1 & 10.5 & 53.6 & 10.1 & 52.7 & 12.8 & 52.4 & 12.9 & 0.95 & $<0.001$ \\
\hline Patients with MetS, $(n)^{\mathrm{b}, \mathrm{c}}$ & $21 / 21$ & & $20 / 21$ & & $22 / 22$ & & $18 / 22$ & & $23 / 23$ & & $20 / 23$ & & & \\
\hline
\end{tabular}

Data are presented as mean $\pm \mathrm{SD}$ unless specified

${ }^{\text {a }}$ Basal DI = HOMA-B/HOMA-IR

${ }^{b}$ IDF criteria was used for MetS classification

${ }^{\mathrm{c}}$ A significant difference was observed between all participants who were diagnosed with MetS pre intervention $(n=66)$ vs post intervention $(n=58$; $p<0.05)$

${ }^{*} p<0.05$ vs baseline

evident in individuals without type 2 diabetes (Table 3 ). Basal DI was significantly increased following only MICT, by $18 \%$ $(p<0.01)$. Moreover, there was no significant difference in fasting glucose and $\mathrm{HbA}_{1 \mathrm{c}}$ levels following all interventions (Table 2).

Insulin sensitivity and beta cell function indices derived from a dynamic state Table 5 presents the change in insulin sensitivity and beta cell function derived from a dynamic state in MetS participants who underwent an OGTT (MICT, $n=14$; 4HIIT, $n=8$; and 1HIIT, $n=9$ ). A significant insulin sensitivity improvement assessed via the Matsuda index was only evident following MICT $(32 \%, p<0.001)$. However, 4HIIT and 1HIIT also showed a trend towards Matsuda index improvement by $17 \%(p=0.26)$ and $28 \%(p=0.16)$, respectively. There was no significant change in first-phase glucosestimulated insulin secretion (GSIS) following all training interventions. Whilst only 1 HIIT showed a significant reduction 
Table 3 Changes in beta cell function and cardiometabolic factors following different types of training in non-type 2 diabetic participants

\begin{tabular}{|c|c|c|c|c|c|c|c|c|c|c|c|c|c|c|}
\hline \multirow[t]{3}{*}{ Variable } & \multicolumn{4}{|c|}{$\operatorname{MICT}(n=15)$} & \multicolumn{4}{|c|}{$4 \mathrm{HIIT}(n=13)$} & \multicolumn{4}{|c|}{$1 \mathrm{HIIT}(n=11)$} & \multicolumn{2}{|c|}{ Between groups } \\
\hline & \multicolumn{2}{|l|}{ Pre } & \multicolumn{2}{|l|}{ Post } & \multicolumn{2}{|l|}{ Pre } & \multicolumn{2}{|l|}{ Post } & \multicolumn{2}{|l|}{ Pre } & \multicolumn{2}{|l|}{ Post } & \multirow[b]{2}{*}{$p$ value } & \multirow[b]{2}{*}{ Effect size } \\
\hline & Mean & SD & Mean & SD & Mean & SD & Mean & SD & Mean & SD & Mean & SD & & \\
\hline \multicolumn{15}{|l|}{ Beta cell function } \\
\hline Basal DI ${ }^{\mathrm{a}}$ & 74.2 & 30.6 & 86.1 & $27.2 *$ & 73.5 & 35.4 & 82.0 & 46.4 & 70.5 & 31.7 & 75.3 & 37.1 & 0.67 & 0.02 \\
\hline Intact proinsulin (pmol/l) & 2.2 & 1.9 & 1.6 & 1.3 & 3.8 & 4.1 & 2.6 & $3.8^{*}$ & 2.1 & 2.2 & 2.4 & 2.4 & 0.28 & 0.06 \\
\hline Intact proinsulin:insulin $\left(\times 10^{-2}\right)$ & 2.3 & 2.4 & 1.9 & 1.6 & 3.1 & 2.9 & 2.3 & $3.2 *$ & 1.9 & 2.0 & 2.5 & 2.5 & 0.35 & 0.05 \\
\hline Intact proinsulin:C-peptide $\left(\times 10^{-3}\right)$ & 1.8 & 1.3 & 1.6 & 1.4 & 3.1 & 3.1 & 2.1 & $2.8^{*}$ & 1.9 & 1.9 & 2.2 & 2.1 & 0.34 & 0.05 \\
\hline \multicolumn{15}{|l|}{ Glucose and insulin metabolism } \\
\hline Fasting glucose (mmol/l) & 5.9 & 1.1 & 5.6 & 0.5 & 6.5 & 3.0 & 6.6 & 2.9 & 6.2 & 0.7 & 6.1 & 0.6 & 0.20 & 0.08 \\
\hline Fasting insulin (pmol/1) & 114 & 67 & 91 & $63 *$ & 108 & 37 & 91 & $44 *$ & 100 & 32 & 95 & 44 & 0.49 & 0.05 \\
\hline C-peptide (nmol/1) & 1.20 & 0.40 & 0.96 & $0.32 *$ & 1.14 & 0.25 & 1.01 & $0.29 *$ & 1.07 & 0.18 & 1.00 & 0.22 & 0.30 & 0.05 \\
\hline $\mathrm{HbA}_{1 \mathrm{c}}, \%(\mathrm{mmol} / \mathrm{mol})$ & 5.7 & 0.5 & 5.6 & 0.4 & 6.2 & 1.6 & 6.2 & 1.8 & 5.8 & 0.4 & 5.9 & 0.7 & 0.65 & 0.10 \\
\hline $\mathrm{HbA}_{1 \mathrm{c}}, \%(\mathrm{mmol} / \mathrm{mol})$ & 38.2 & 5.4 & 37.3 & 4.5 & 44.2 & 17.6 & 44.4 & 19.4 & 39.0 & 4.7 & 40.5 & 7.0 & 0.65 & 0.10 \\
\hline HOMA-IR (insulin) & 2.2 & 1.2 & 1.7 & $1.1^{*}$ & 2.2 & 1.0 & 1.8 & $0.9^{*}$ & 1.9 & 0.6 & 1.8 & 0.9 & 0.45 & 0.05 \\
\hline HOMA-B (insulin) & 117.8 & 51.1 & 109.4 & 52.4 & 109.2 & 36.8 & 93.8 & $32.2 *$ & 98.3 & 27.9 & 96.3 & 37.2 & 0.74 & 0.02 \\
\hline HOMA-B (C-peptide) & 137.2 & 31.8 & 125.7 & 33.6 & 131.3 & 33.5 & 116.4 & $32.2 *$ & 120 & 23.3 & 116 & 28.4 & 0.47 & 0.04 \\
\hline \multicolumn{15}{|l|}{ CRF } \\
\hline Absolute $\dot{V} \mathrm{O}_{2 \text { peak }}(1 / \mathrm{min})$ & 2.7 & 0.8 & 2.8 & 0.8 & 2.4 & 0.7 & 2.7 & 1.0 & 2.2 & 0.8 & 2.3 & 0.9 & 0.37 & 0.06 \\
\hline Relative $\dot{V} \mathrm{O}_{2 \text { peak }}\left(\mathrm{ml} \mathrm{kg}^{-1} \mathrm{~min}^{-1}\right)$ & 27.9 & 7.6 & 28.9 & 6.9 & 24.5 & 4.5 & 28.4 & $6.8^{*}$ & 24.3 & 6.1 & 26.0 & 6.3 & 0.46 & 0.04 \\
\hline \multicolumn{15}{|c|}{ MetS risk factors and anthropometric measures } \\
\hline HDL-C (mmol/l) & 1.21 & 0.35 & 1.20 & 0.33 & 0.97 & 0.28 & 1.07 & 0.30 & 1.43 & 0.42 & 1.47 & 0.44 & 0.57 & 0.03 \\
\hline Triacylglycerol (mmol/l) & 1.67 & 0.81 & 1.70 & 1.05 & 2.40 & 1.41 & 2.14 & 1.15 & 1.81 & 0.65 & 1.60 & 0.53 & 0.89 & 0.01 \\
\hline SBP (mmHg) & 131 & 15 & 124 & 10 & 134 & 12 & 127 & $11 *$ & 133 & 14 & 121 & $8^{*}$ & 0.21 & 0.05 \\
\hline DBP (mmHg) & 87 & 11 & 82 & 8 & 87 & 7 & 82 & $8 *$ & 83 & 8 & 79 & 4 & 0.83 & 0.01 \\
\hline WC & 107 & 14 & 105 & 14 & 105 & 11 & 104 & 9 & 102 & 15 & 98 & 13 & 0.30 & 0.06 \\
\hline Waist:hip ratio & 0.92 & 0.09 & 0.90 & 0.08 & 0.90 & 0.08 & 0.89 & 0.06 & 0.91 & 0.10 & 0.88 & 0.07 & 0.37 & 0.03 \\
\hline Weight (kg) & 99 & 21 & 98 & 22 & 97 & 18 & 96 & 18 & 91 & 22 & 89 & 21 & 0.77 & 0.01 \\
\hline $\mathrm{BF}(\%)$ & 40.8 & 9.4 & 40.8 & 9.6 & 43.1 & 5.1 & 42.8 & 5.5 & 43.3 & 6.1 & 42.6 & 6.2 & 0.74 & 0.02 \\
\hline $\mathrm{TF}(\%)$ & 43.8 & 9.0 & 43.7 & 9.0 & 45.2 & 4.4 & 45.2 & 4.8 & 45.7 & 5.4 & 45.0 & 5.0 & 0.81 & 0.01 \\
\hline FFM (kg) & 55 & 13 & 55 & 13 & 53 & 11 & 52 & 10 & 49 & 14 & 49 & 14 & 0.45 & 0.04 \\
\hline
\end{tabular}

Data are presented as mean $\pm \mathrm{SD}$

${ }^{\mathrm{a}}$ Basal DI = HOMA-B/HOMA-IR

${ }^{*} p<0.05$ vs baseline

in second-phase GSIS, by $-20 \%(p=0.04)$, 4HIIT also showed a trend towards a reduction $(-22 \%, p=0.42)$. There were no significant changes in first- or second-phase DI, $2 \mathrm{~h}$ glucose or insulin or total AUC glucose or insulin following any of the training interventions.

CRF and MVPA CRF (relative $\dot{V} \mathrm{O}_{2 \text { peak }}\left[\mathrm{ml} / \mathrm{kg}^{-1} \mathrm{~min}^{-1}\right]$ ) was significantly improved in only the HIIT groups, with increases of $15 \%, 8 \%$ and $3 \%$ following 4HIIT, 1HIIT and MICT, respectively. There was also a small to medium group $\times$ time interaction effect on $\mathrm{CRF}$, indicating a greater capacity of the HIIT groups to improve CRF compared with MICT, $\left[\mathrm{F}(2,59)=1.99, p=0.15, \eta^{2}=0.06\right]$ (Table 2).
MVPA time (min/day) also significantly increased following all training interventions (MICT, 47\%; 4HIIT, 33\%; 1HIIT, 26\%; group difference, $p=0.03, \eta^{2}=0.12$ ). Interestingly, only the 4HIIT group improved CRF despite a significant increase in MVPA following both MICT $(49 \%, p<0.001)$ and 4HIIT $(44 \%, p=0.02)$ in non-type 2 diabetic individuals.

\section{MetS risk factors and anthropometric measurements} Table 2 presents changes in MetS risk factors and anthropometric measurements from pre to post intervention in all participants. $\mathrm{BP}$ and $\mathrm{WC}$ were only significantly reduced following MICT (SBP $-5 \%$; DBP $-6 \%$; WC $-2 \%$ ) and $1 \mathrm{HIIT}$ (SBP 
Table 4 Changes in beta cell function and cardiometabolic factors following different types of training in type 2 diabetic participants

\begin{tabular}{|c|c|c|c|c|c|c|c|c|c|c|c|c|c|c|}
\hline \multirow[t]{3}{*}{ Variable } & \multicolumn{4}{|c|}{$\operatorname{MICT}(n=6)$} & \multicolumn{4}{|c|}{ 4HIIT $(n=9)$} & \multicolumn{4}{|c|}{ 1HIIT $(n=12)$} & \multicolumn{2}{|c|}{ Between groups } \\
\hline & \multicolumn{2}{|l|}{ Pre } & \multicolumn{2}{|l|}{ Post } & \multicolumn{2}{|l|}{ Pre } & \multicolumn{2}{|l|}{ Post } & \multicolumn{2}{|l|}{ Pre } & \multicolumn{2}{|l|}{ Post } & \multirow[b]{2}{*}{$p$ value } & \multirow[b]{2}{*}{ Effect siz } \\
\hline & Mean & SD & Mean & SD & Mean & SD & Mean & $\mathrm{SD}$ & Mean & $\mathrm{SD}$ & Mean & $\mathrm{SD}$ & & \\
\hline \multicolumn{15}{|l|}{ Beta cell function } \\
\hline Basal DI ${ }^{\mathrm{a}}$ & 60.0 & 42.7 & 73.6 & $45.2^{*}$ & 45.4 & 25.0 & 44.4 & 18.2 & 49.0 & 22.7 & 58.3 & 44.0 & 0.53 & 0.05 \\
\hline Intact proinsulin (pmol/1) & 5.4 & 5.8 & 4.9 & 4.4 & 4.7 & 3.4 & 4.7 & 4.0 & 6.4 & 4.9 & 9.2 & 9.2 & 0.35 & 0.09 \\
\hline Intact proinsulin:insulin $\left(\times 10^{-2}\right)$ & 6.4 & 7.9 & 8.0 & 8.9 & 4.5 & 3.1 & 4.9 & 3.0 & 6.1 & 3.8 & 9.5 & 8.4 & 0.45 & 0.07 \\
\hline Intact proinsulin:C-peptide $\left(\times 10^{-3}\right)$ & 4.8 & 5.6 & 5.3 & 5.4 & 4.1 & 3.0 & 4.5 & 3.2 & 5.2 & 3.0 & 7.3 & 6.1 & 0.49 & $<0.001$ \\
\hline \multicolumn{15}{|l|}{ Glucose and insulin metabolism } \\
\hline Fasting glucose $(\mathrm{mmol} / \mathrm{l})$ & 7.3 & 1.4 & 6.8 & 1.1 & 7.9 & 1.7 & 8.2 & 2.9 & 7.8 & 2.0 & 7.9 & 2.3 & 0.54 & 0.05 \\
\hline Fasting insulin (pmol/1) & 116 & 81 & 84 & 47 & 103 & 43 & 92 & 34 & 113 & 72 & 102 & 80 & 0.56 & 0.04 \\
\hline C-peptide (nmol/1) & 1.21 & 0.31 & 1.00 & $0.27 *$ & 1.15 & 0.31 & 1.04 & 0.37 & 1.17 & 0.37 & 1.13 & 0.50 & 0.44 & 0.07 \\
\hline $\mathrm{HbA}_{1 \mathrm{c}},(\%)$ & 6.2 & 0.9 & 6.0 & 0.7 & 7.6 & 2.8 & 7.6 & 3.0 & 7.0 & 1.4 & 6.7 & 0.8 & 0.46 & 0.06 \\
\hline $\mathrm{HbA}_{1 \mathrm{c}}, \mathrm{mmol} / \mathrm{mol}$ & 44.4 & 10.5 & 42.6 & 7.1 & 59.7 & 30.4 & 60.0 & 33.1 & 53.2 & 15.4 & 49.3 & 8.2 & 0.46 & 0.06 \\
\hline HOMA-IR (insulin) & 2.3 & 1.6 & 1.7 & 0.9 & 2.1 & 0.8 & 1.9 & 0.6 & 2.3 & 1.4 & 2.1 & 1.5 & 0.49 & 0.05 \\
\hline HOMA-B (insulin) & 75.9 & 23.9 & 71.4 & 24.6 & 69.5 & 35.8 & 67.3 & 34.2 & 74.2 & 47.8 & 64.2 & 43.5 & 0.67 & 0.03 \\
\hline HOMA-B (C-peptide) & 99.8 & 34.7 & 97.4 & 28.7 & 88.5 & 39.4 & 85.7 & 42.5 & 90.4 & 37.9 & 87.0 & 36.7 & 0.87 & 0.01 \\
\hline \multicolumn{15}{|l|}{ CRF } \\
\hline Absolute $\dot{V} \mathrm{O}_{2 \text { peak }}(1 / \mathrm{min})$ & 2.7 & 0.6 & 2.7 & 0.4 & 2.4 & 0.7 & 2.7 & 0.8 & 2.4 & 0.6 & 2.6 & $0.6^{*}$ & 0.37 & 0.08 \\
\hline Relative $\dot{V} \mathrm{O}_{2 \text { peak }}\left(\mathrm{ml} \mathrm{kg}^{-1} \min ^{-1}\right)$ & 27.9 & 7.9 & 28.3 & 6.9 & 24.6 & 7.4 & 27.9 & 8.3 & 27.3 & 6.8 & 29.7 & $6.8^{*}$ & 0.30 & 0.10 \\
\hline \multicolumn{15}{|l|}{ MetS risk factors \& anthropometric measures } \\
\hline HDL-C (mmol/l) & 0.94 & 0.29 & 1.23 & 0.59 & 1.07 & 0.41 & 1.12 & 0.46 & 0.89 & 0.23 & 1.10 & $0.24 *$ & 0.40 & 0.07 \\
\hline Triacylglycerol (mmol/l) & 1.87 & 0.64 & 1.43 & 0.41 & 2.15 & 0.89 & 2.23 & 1.30 & 1.90 & 0.76 & 1.73 & 0.65 & 0.34 & 0.08 \\
\hline Systolic BP (mmHg) & 134 & 9 & 127 & $12 *$ & 121 & 7 & 127 & 8 & 136 & 12 & 130 & $16^{*}$ & 0.03 & 0.24 \\
\hline Diastolic BP (mmHg) & 86 & 5 & 79 & $6^{*}$ & 80 & 7 & 78 & 7 & 82 & 6 & 80 & 9 & 0.89 & 0.01 \\
\hline $\mathrm{WC}(\mathrm{cm})$ & 108 & 10 & 105 & 8 & 106 & 11 & 104 & 11 & 104 & 11 & 102 & $11^{*}$ & 0.77 & 0.02 \\
\hline Waist:hip ratio & 0.99 & 0.09 & 0.96 & $0.09 *$ & 0.91 & 0.09 & 0.91 & 0.09 & 0.97 & 0.06 & 0.94 & 0.06 & 0.59 & 0.03 \\
\hline Weight $(\mathrm{kg})$ & 100 & 10 & 97 & 12 & 100 & 16 & 98 & 17 & 93 & 20 & 92 & 20 & 0.66 & 0.04 \\
\hline $\mathrm{BF}(\%)$ & 34.2 & 4.7 & 32.8 & 4.1 & 41.1 & 8.3 & 40.2 & 9.1 & 35.7 & 6.6 & 35.4 & 6.9 & 0.55 & 0.06 \\
\hline $\mathrm{TF}(\%)$ & 38.7 & 6.1 & 37.0 & 5.5 & 44.4 & 7.2 & 43.4 & 8.0 & 40.0 & 6.3 & 39.7 & 6.3 & 0.56 & 0.05 \\
\hline FFM (kg) & 62 & 6 & 61 & 6 & 56 & 9 & 56 & 9 & 56 & 10 & 56 & 11 & 0.24 & 0.13 \\
\hline
\end{tabular}

Data are presented as mean \pm SD unless specified

${ }^{\text {a }}$ Basal DI = HOMA-B/HOMA-IR

${ }^{*} p<0.05$ vs baseline

$-7 \%$; DBP $-4 \%$; $\mathrm{WC}-3 \%$ ), with a small to medium group $\times$ time interaction effect size, indicating a greater capacity for these training interventions to reduce these MetS risk factors when compared with 4HIIT. Only 1HIIT significantly increased HDL-cholesterol (HDL-C) from pre to post intervention (11\%), but MICT (7\%) and 4HIIT (8\%) also showed a trend towards an increase. There were no other significant changes in the lipid profile. Interestingly, in a subanalysis of participants with and without type 2 diabetes (Tables 2 and 3), 1HIIT was still shown to be sufficient to improve SBP, WC and HDL-C. Moreover, eight out of the 66 individuals included in the trial were no longer diagnosed with MetS $(p<0.01)$ following the 16 week intervention, and seven of these eight were from the HIIT groups (Table 2). There were no significant changes in weight and body composition (body fat $\%$ $[\mathrm{BF} \%]$, trunk fat $\%[\mathrm{TF} \%]$ and free fat mass [FFM]) following all training interventions (Tables 2, 3 and 4). Moreover, there were no significant within- and between-group changes in total energy intake from pre to post exercise training.

Mixed linear model regression analysis The mechanism by which 4HIIT could significantly reduce fasting intact proinsulin concentration indices in non-type 2 diabetic individuals was further examined via the mixed linear model regression analysis. The decrease in intact proinsulin concentration indices following 4HIIT was significantly associated with the change in glucose 
Table 5 Change in beta cell function and glucose and insulin metabolism indices derived from an OGTT

\begin{tabular}{|c|c|c|c|c|c|c|c|c|c|c|c|c|c|c|}
\hline \multirow[t]{4}{*}{ Variable } & \multirow{2}{*}{\multicolumn{4}{|c|}{$\begin{array}{l}\text { MICT }(n=14) \\
\begin{array}{l}\text { NGT, } n=4 ; \mathrm{IFG} / \mathrm{IGT} / \mathrm{T} 2 \mathrm{D}, \\
n=10)\end{array}\end{array}$}} & \multirow{2}{*}{\multicolumn{4}{|c|}{$\begin{array}{l}4 \mathrm{HIIT}(n=8) \\
\begin{array}{l}(\mathrm{NGT}, n=2 ; \mathrm{IFG} / \mathrm{IGT} / \mathrm{T} 2 \mathrm{D}, \\
n=6)\end{array}\end{array}$}} & \multicolumn{4}{|c|}{ 1HIIT ( $n=9)$} & \multicolumn{2}{|c|}{ Between groups } \\
\hline & & & & & & & & & \multicolumn{4}{|c|}{$\begin{array}{l}\text { (NGT, } n=0 ; \text { IFG/IGT/T2D, } \\
n=9)\end{array}$} & & \\
\hline & \multicolumn{2}{|l|}{ Pre } & \multicolumn{2}{|l|}{ Post } & \multicolumn{2}{|l|}{ Pre } & \multicolumn{2}{|l|}{ Post } & \multicolumn{2}{|l|}{ Pre } & \multicolumn{2}{|l|}{ Post } & & \\
\hline & Mean & SD & Mean & SD & Mean & SD & Mean & SD & Mean & SD & Mean & SD & $p$ value & Effect size \\
\hline \multicolumn{15}{|l|}{ Beta cell function } \\
\hline First-phase DI ${ }^{\mathrm{a}}$ & 0.8 & 0.6 & 0.9 & 0.4 & 0.7 & 1.0 & 0.7 & 0.7 & 0.3 & 0.1 & 0.3 & 0.2 & 0.06 & 0.12 \\
\hline Second-phase DI ${ }^{\mathrm{b}}$ & 1.3 & 0.8 & 1.5 & 0.7 & 1.1 & 1.1 & 1.2 & 0.9 & 0.6 & 0.3 & 0.6 & 0.3 & 0.08 & 0.16 \\
\hline \multicolumn{15}{|l|}{ Glucose and insulin metabolism } \\
\hline $2 \mathrm{~h}$ glucose $(\mathrm{mmol} / \mathrm{l})$ & 8.2 & 3.9 & 7.9 & 4.0 & 11.5 & 6.3 & 10.2 & 7.0 & 14.1 & 5.7 & 14.1 & 5.2 & 0.50 & 0.04 \\
\hline $2 \mathrm{~h}$ insulin $(\mathrm{pmol} / \mathrm{l})$ & 670.3 & 707.0 & 448.2 & $430.4 *$ & 893.5 & 706.7 & 443.6 & 247.9 & 770.7 & 836.7 & 566.2 & 491.8 & 0.47 & 0.03 \\
\hline Total glucose AUC $(\mathrm{mmol} / 1 \times \min )$ & 18 & 6 & 16 & 3 & 24 & 8 & 22 & 10 & 28 & 7 & 28 & 8 & 0.13 & 0.12 \\
\hline Total insulin AUC $(\mathrm{pmol} / 1 \times \mathrm{min})$ & 1309 & 854 & 1178 & 749 & 1850 & 1679 & 1254 & 717 & 1463 & 1158 & 1112 & 812 & 0.67 & 0.03 \\
\hline Matsuda index & 2.2 & 1.2 & 2.9 & $1.5^{*}$ & 1.8 & 0.8 & 2.1 & 0.5 & 1.8 & 1.0 & 2.3 & 1.7 & 0.42 & 0.05 \\
\hline $\begin{array}{l}\text { First-phase glucose-stimulated } \\
\text { insulin release }\end{array}$ & 0.4 & 0.2 & 0.4 & 0.2 & 0.6 & 1.1 & 0.4 & 0.3 & 0.2 & 0.2 & 0.2 & 0.2 & 0.28 & 0.07 \\
\hline $\begin{array}{l}\text { Second-phase glucose-stimulated } \\
\text { insulin release } \mathrm{e}^{\mathrm{d}}\end{array}$ & 0.7 & 0.5 & 0.7 & 0.4 & 0.9 & 1.1 & 0.7 & 0.5 & 0.5 & 0.5 & 0.4 & $0.3^{*}$ & 0.50 & 0.05 \\
\hline
\end{tabular}

Data are presented as mean $\pm \mathrm{SD}$

${ }^{\text {a }}$ First-phase $\mathrm{DI}=\left(\left[\mathrm{I}_{0-30} / \mathrm{G}_{0-30}\right] \times\right.$ Matsuda index $)$

${ }^{\mathrm{b}}$ Second-phase $\mathrm{DI}=\left(\mathrm{I}_{60-120} / \mathrm{G}_{60-120}\right) \times$ Matsuda index

${ }^{\mathrm{c}}$ First-phase glucose-stimulated insulin release $=\mathrm{I}_{0-30} / \mathrm{G}_{0-30}$

${ }^{\mathrm{d}}$ Second-phase glucose-stimulated insulin release $=\mathrm{I}_{60-120} / \mathrm{G}_{60-120}$

* Significantly different from baseline, $p<0.05$

NGT, normal glucose tolerance; T2D, type 2 diabetes

concentration (proinsulin, $p<0.01$; proinsulin:insulin, $p<0.001$; proinsulin:C-peptide, $p<0.005$ ) and HOMA-IR (proinsulin, $p<0.01$; proinsulin:insulin, $p=0.02$; proinsulin:C-peptide, $p<0.001)$. There was also a trend towards an association between a reduction in intact proinsulin concentration indices from pre to post intervention and the change in triacyglycerol concentration (proinsulin, $p=0.08$; proinsulin:insulin, $p=0.05$; proinsulin:C-peptide, $p=0.05$ ), but not HDL-C (proinsulin, $p=0.92$; proinsulin:insulin, $p=0.90$; proinsulin:C-peptide, $p=0.98$ ). We also found a significant association between the decrease in intact proinsulin indices and the changes in BF\% (proinsulin, $p<0.001$; proinsulin:C-peptide, $p=0.02$ ) and TF\% (proinsulin, $p<0.001$; proinsulin:insulin, $p=0.03$; proinsulin: C-peptide, $p<0.01$ ), but not CRF (proinsulin, $p=0.43$; proinsulin:insulin, $p=0.78$; proinsulin:C-peptide, $p=0.90$ ). Interestingly, the change in body fat indices was significantly dependent on the change in $\mathrm{CRF}$ (BF\%, $p<0.001$; TF\%, $p<0.005$ ), but not MVPA (BF\%, $p=0.47$; TF\%, $p=0.51$ ).

\section{Discussion}

This is the first randomised trial to compare the impact of different volumes of HIIT (4HIIT and 1HIIT) and MICT on insulin quality in MetS individuals. The main finding is that high-volume HIIT (4HIIT) was the only potent stimulus to enhance insulin quality, depicted by a reduction of fasting intact proinsulin concentration indices in MetS individuals without type 2 diabetes. Interestingly, these fasting intact proinsulin concentration indices did not change from pre to post intervention in MetS individuals with type 2 diabetes. Our study therefore provides evidence that combining increased intensity and volume of training, predominantly aerobic exercise, may be important for improving insulin quality in MetS individuals who have not progressed to type 2 diabetes.

The significant decrease in fasting intact proinsulin concentration indices following 4HIIT in non-type 2 diabetic participants did not translate to a significant improvement in glycaemic control $\left(\mathrm{HbA}_{1 \mathrm{c}}\right.$ and fasting glucose). However, the improvement in fasting intact proinsulin concentration indices could be deemed clinically significant, given that people without type 2 diabetes with a low concentration of fasting intact proinsulin have been shown to be less susceptible to developing type 2 diabetes [8], ultimately reducing risk of all-cause and CV mortality $[9,10]$. Based on a previous study [27], it could be inferred that the improvement in fasting intact proinsulin concentration following 4HIIT could be solely attributed to the exercise dose, independent of a change in dietary intake. Torjesen et al [27] showed a significant reduction in fasting intact proinsulin following an exercise-only 
intervention, with no significant difference in improvement compared with exercise plus diet change.

The 4HIIT-induced improvement in fasting intact proinsulin concentration indices was likely due to a reversal of glucolipotoxicity in pancreatic beta cells [28] and CRF improvement-related metabolic adaptations such as mitochondrial biogenesis (i.e. peroxisome proliferator-activated receptor- $\gamma$ coactivator 1 , alpha $[$ PGC- $1 \alpha]$ ) that are associated with increased antioxidant defence and insulin sensitivity [29, 30]. This is supported by our finding, which showed that the increase in intact proinsulin concentration indices is associated with detrimental changes in glucose, HOMA-IR, triacylglycerol and body fat indices, which are all contributing factors to a glucolipotoxic environment. Increased CRF has been postulated to be an antidote against traditional CVD risk factors [12], which are all contributing factors to a glucolipotoxic environment and thus oxidative stress-induced impaired beta cell function [3]. The transient reactive oxygen species (ROS) produced via the mitochondrial metabolism of glucose are known to act as a coupling factor to promote beta cell insulin secretion [31]. However, a glucolipotoxic milieu-induced excessive and continuous ROS production in an IR state makes pancreatic beta cells susceptible to oxidative damage because of the relatively low expression of antioxidant enzymes [32]. This, in turn, leads to ER stress [33, 34] and thus incomplete processing of proinsulin to produce higher-quality insulin.

The glucolipotoxic environment-induced oxidative stress could, however, be buffered by the upregulation of PGC- $1 \alpha$ that usually occurs following HIIT [35-38] promoting expression of antioxidant enzymes [39]. Specifically, Tjonna et al [35] demonstrated $138 \%$ increase in PGC- $1 \alpha$ following 16 weeks of 4HIIT (three times/week), accompanied by a significant improvement in vascular function and reversal of MetS risk factors in individuals with MetS. This notion is further supported by studies that showed HIIT to significantly improve vascular function $[40,41]$ and diabetes-related inflammatory cytokines [42], which are factors related to reduced oxidative stressinduced pancreatic beta cell dysfunction.

Our results also suggest that the exercise HIIT volume matters in promoting beta cell function improvement, given that 1 HIIT did not significantly reduce fasting intact proinsulin concentration indices despite also improving CRF. This is supported by a previous study, which suggests that higher exercise doses are necessary to improve beta cell function in adults with impaired glucose tolerance (IGT) and/or impaired fasting glucose (IFG) [43]. The importance of exercise volume is further highlighted by our results, which showed a slight nonsignificant increase in intact proinsulin indices in the 1HIIT group who were non-type 2 diabetic (Table 3), which is further amplified in patients with type 2 diabetes following the intervention (Table 4). This suggests that this low-volume 1HIIT protocol may not be adequate to maintain pancreatic beta cell function or to deter the progression of type 2 diabetes.
A potential additive mechanism by which high-volume HIIT exercise could significantly decrease fasting intact proinsulin concentration could be a change in pancreatic gene expression associated with insulin synthesis and GSIS. Indeed, Koranyi et al [44] found a significant decrease in pancreatic glucokinase and proinsulin mRNA levels in rodents following 3 weeks of running at $25 \mathrm{~m} / \mathrm{min}$ up a $15 \%$ incline for $90 \mathrm{~min} /$ day for 6 days/week. In contrast, Slentz et al [45] demonstrated that a lower exercise dose of $1220 \mathrm{kcal} /$ week is sufficient to improve pancreatic beta cell function in sedentary overweight adults, perhaps because of an increase in insulin sensitivity that has been shown to be notable even following a small exercise dose of $400 \mathrm{kcal} /$ week [46]. The discrepancies between results may be attributed to the methods of pancreatic beta cell function measures employed between studies.

Perhaps a greater exercise stimulus, whether it may be the type (e.g. resistance training) or duration of the exercise programme ( $>16$ weeks), may be required to provoke enhancement of insulin quality in MetS individuals with type 2 diabetes. For example, Church et al [47] showed that in type 2 diabetic patients, a combination of resistance and aerobic exercise for 9 months was necessary to induce a significant improvement in glycaemic control $\left(\mathrm{HbA}_{1 \mathrm{c}}\right)$, which could infer a parallel improvement in insulin quality. In the same study, type 2 diabetic patients who only performed aerobic or resistance training of similar time duration per week ( $140 \mathrm{~min} /$ week) as the combination training group, showed no significant change in $\mathrm{HbA}_{1 \mathrm{c}}$. This is consistent with our study wherein no significant improvement in either fasting intact proinsulin concentration or $\mathrm{HbA}_{1 \mathrm{c}}$ was evident in a subgroup of MetS participants with type 2 diabetes following any of the exercise interventions. In contrast, other studies have demonstrated a significant $\mathrm{HbA}_{1 \mathrm{c}}$ improvement following 8-12 weeks of HIIT in type 2 diabetic patients [48, 49], which could be attributed to the difference in pre-existing glycaemic status of individuals within each study. Indeed, Malin et al [50] showed that individuals with a combination of IFG and IGT were less responsive to exercise-induced improvement in insulin sensitivity relative to individuals with IGT alone at baseline.

Alternatively, it could be speculated that the lack of improvement in fasting intact proinsulin concentration following the interventions in MetS patients with type 2 diabetes could be due to the intake of metformin medication by the majority of type 2 diabetic patients included in this trial. Metformin has been shown to blunt the full effect of exercise on insulin sensitivity, perhaps through its opposing effect on ROS signalling, downregulating cellular signals involved with mitochondrial biogenesis, endothelial function (nitric oxide-mediated blood flow) and glucose uptake (GLUT4) [51] that could indirectly affect an insulin quality improvement and thus beta cell function. Malin and colleagues [52] showed that 12 weeks of treatment with an exercise-only programme increased 
insulin sensitivity by $25-30 \%$ compared with a combination of the same exercise programme and metformin intake or metformin only. Similarly, our study showed a significant improvement in insulin sensitivity (14\% decrease in HOMA-IR) following 4HIIT in MetS individuals without type 2 diabetes, but not in patients with type 2 diabetes, of whom $\sim 70 \%$ were taking metformin.

Inconsistent with recent studies showing improved firstphase DI following HIIT but not MICT in type 2 diabetic patients $[48,53]$, our study only showed a significant basal DI improvement following MICT in MetS individuals with type 2 diabetes. This improvement resulted from increased insulin sensitivity with no accompanying change in insulin secretion. Slentz et al [45] also showed a significant increase in first-phase DI following 8 months of a similar dose of MICT (40-55\% $\dot{V} \mathrm{O}_{2 \text { peak }}, \sim 200 \mathrm{~min} /$ week) in sedentary overweight adults, albeit through an additional mechanism, showing an improvement in both insulin sensitivity and insulin secretion. The discrepancies between results could be attributed to the difference in HIIT and MICT dose used in the studies. Alternatively, it could also be argued that the DI measure becomes unreliable when comparing beta cell function changes following regular exercise between studies of different cohorts. This is because the change in insulin secretion that accompanies a change in insulin sensitivity following regular exercise is highly dependent on the state of the individual's beta cell function prior to the intervention. It has been reported that individuals with healthy beta cell function demonstrate reduced insulin secretion concomitant with improved insulin sensitivity following regular exercise. In contrast, individuals with impaired beta cell function demonstrate augmented insulin secretion with improved insulin sensitivity [54-56].

Limitations The mere assessment of fasting intact proinsulin concentration at a steady-state condition has been criticised as providing only limited physiological information of beta cell function relative to markers derived from dynamic conditions [7]. However, a recent study showed fasting intact proinsulin concentration to be a better predictor of both worsening of hyperglycaemia and incidence of type 2 diabetes compared with glucose-stimulated intact proinsulin concentration assessed via an OGTT in 9396 individuals without type 2 diabetes from recruitment [8]. Moreover, the variation in the time of metformin intake $(\mathrm{am} / \mathrm{pm})$ at both intervention time points could have been the source of variance seen in our results. However, it should be noted that the drug was taken at approximately the same time of day by each participant at both intervention timepoints, thereby decreasing the chance that it would have affected our overall results.

Conclusion Higher-volume HIIT (4HIIT) decreased intact proinsulin levels in MetS participants without type 2 diabetes, suggesting that this higher-intensity volume of exercise improved beta cell function. This, therefore, provides evidence that at least 16 weeks of a combination of higher exercise intensity and volume, as seen with 4HIIT, may be important in improving insulin quality in MetS individuals without type 2 diabetes. However, it should be noted that this did not translate to a significant improvement in glycaemic control $\left(\mathrm{HbA}_{1 \mathrm{c}}\right.$ and fasting glucose). Nonetheless, the intact proinsulin concentration reduction evident following 4HIIT could be deemed clinically relevant given that people without type 2 diabetes with low fasting intact proinsulin concentration have been reported to be less susceptible to developing type 2 diabetes [8] and thus lower risk of all-cause and CV mortality [9, 10]. More studies are, however, warranted to investigate whether a greater exercise stimulus may be required to enhance insulin quality in MetS individuals with type 2 diabetes.

Acknowledgements Gary Wilson (School of Human Movement and Nutrition Sciences, The University of Queensland, Australia) helped with laboratory analysis of intact proinsulin, insulin, C-peptide and glucose.

Contribution statement JSR wrote the manuscript, collected and analysed/interpreted the data and reviewed/edited and approved the final draft of the manuscript. JSR and JSC designed the study. JSC, LCD, FB, ARM, BC, SEK and RGF also substantially contributed to the conception and design, analysis and interpretation of data and reviewed/edited and approved the final draft of the manuscript. JSC is the guarantor of this study.

Funding Funding for this study was provided by the Norwegian University of Science and Technology and from an unrestricted research grant from the Coca-Cola company. Funding for the collection of physical activity data was derived from a 'UQ New Staff Start Up' grant awarded to $\mathrm{BC}$.

Duality of interest The authors declare that there is no duality of interest associated with this manuscript.

\section{References}

1. Grundy SM, Brewer HB Jr, Cleeman JI, Smith SC Jr, Lenfant C (2004) Definition of metabolic syndrome: report of the National Heart, Lung, and Blood Institute/American Heart Association conference on scientific issues related to definition. Arterioscler Thromb Vasc Biol 24:e13-e18

2. Malin SK, Finnegan S, Fealy CE, Filion J, Rocco MB, Kirwan JP (2014) beta-Cell dysfunction is associated with metabolic syndrome severity in adults. Metab Syndr Relat Disord 12:79-85

3. Poitout V, Robertson RP (2008) Glucolipotoxicity: fuel excess and beta-cell dysfunction. Endocr Rev 29:351-366

4. Rhodes CJ, Alarcon C (1994) What beta-cell defect could lead to hyperproinsulinemia in NIDDM? Some clues from recent advances made in understanding the proinsulin-processing mechanism. Diabetes 43:511-517

5. Alarcon C, Leahy JL, Schuppin GT, Rhodes CJ (1995) Increased secretory demand rather than a defect in the proinsulin conversion mechanism causes hyperproinsulinemia in a glucose-infusion rat 
model of non-insulin-dependent diabetes mellitus. J Clin Invest 95: 1032-1039

6. Pfutzner A, Forst T (2011) Elevated intact proinsulin levels are indicative of Beta-cell dysfunction, insulin resistance, and cardiovascular risk: impact of the antidiabetic agent pioglitazone. J Diabetes Sci Technol 5:784-793

7. Cersosimo E, Solis-Herrera C, Trautmann ME, Malloy J, Triplitt CL (2014) Assessment of pancreatic beta-cell function: review of methods and clinical applications. Curr Diabetes Rev 10:2-42

8. Vangipurapu J, Stancakova A, Kuulasmaa T, Kuusisto J, Laakso M (2015) Both fasting and glucose-stimulated proinsulin levels predict hyperglycemia and incident type 2 diabetes: a population-based study of 9,396 finnish men. PLoS One 10:e0124028

9. Alssema M, Dekker JM, Nijpels G, Stehouwer CD, Bouter LM, Heine RJ (2005) Proinsulin concentration is an independent predictor of all-cause and cardiovascular mortality: an 11-year follow-up of the Hoorn Study. Diabetes Care 28:860-865

10. Zethelius B, Byberg L, Hales CN, Lithell H, Berne C (2002) Proinsulin is an independent predictor of coronary heart disease: report from a 27-year follow-up study. Circulation 105:2153-2158

11. Haffner SM, Mykkanen L, Valdez RA et al (1994) Disproportionately increased proinsulin levels are associated with the insulin resistance syndrome. J Clin Endocrinol Metab 79: 1806-1810

12. Blair SN, Kampert JB, Kohl HW 3rd et al (1996) Influences of cardiorespiratory fitness and other precursors on cardiovascular disease and all-cause mortality in men and women. JAMA 276:205210

13. Pattyn N, Cornelissen VA, Eshghi SR, Vanhees L (2013) The effect of exercise on the cardiovascular risk factors constituting the metabolic syndrome: a meta-analysis of controlled trials. Sports Med (Auckland, NZ) 43:121-133

14. Bateman LA, Slentz CA, Willis LH et al (2011) Comparison of aerobic versus resistance exercise training effects on metabolic syndrome (from the Studies of a Targeted Risk Reduction Intervention Through Defined Exercise - STRRIDE-AT/RT). Am J Cardiol 108: $838-844$

15. Johnson JL, Slentz CA, Houmard JA et al (2007) Exercise training amount and intensity effects on metabolic syndrome (from Studies of a Targeted Risk Reduction Intervention through Defined Exercise). Am J Cardiol 100:1759-1766

16. Trost SG, Owen N, Bauman AE, Sallis JF, Brown W (2002) Correlates of adults' participation in physical activity: review and update. Med Sci Sports Exerc 34:1996-2001

17. Weston KS, Wisloff U, Coombes JS (2014) High-intensity interval training in patients with lifestyle-induced cardiometabolic disease: a systematic review and meta-analysis. Br J Sports Med 48:12271234

18. Jelleyman C, Yates T, O'Donovan G et al (2015) The effects of high-intensity interval training on glucose regulation and insulin resistance: a meta-analysis. Obes Rev 16:942-961

19. Tjonna AE, Leinan IM, Bartnes AT et al (2013) Low- and highvolume of intensive endurance training significantly improves maximal oxygen uptake after 10 -weeks of training in healthy men. PLoS One 8:e65382

20. Solomon TP, Malin SK, Karstoft K et al (2015) Association between cardiorespiratory fitness and the determinants of glycemic control across the entire glucose tolerance continuum. Diabetes Care 38:921-929

21. Ramos JS, Dalleck LC, Ramos MV et al (2016) Twelve minutes/ week of high-intensity exercise significantly decreases aortic reservoir pressure in individuals with metabolic syndrome. J Hypertens (in press)

22. Ramos JS, Ramos MV, Dalleck LC, et al. (2016) Fitness is independently associated with central hemodynamics in metabolic syndrome. Med Sci Sports Exerc. doi:10.1249/MSS.0000000000000916
23. University of Oxford. Diabetes Trials Unit - The Oxford Centre for Diabetes, Endocrinology and Metabolism. Available from http://www.dtu.ox.ac.uk, accessed 8 Jan 2016

24. American College of Sports Medicine (2014) ACSM's guidelines for exercise testing and prescription. Lippincott Williams \& Wilkins, Baltimore

25. Ryan CG, Grant PM, Tigbe WW, Granat MH (2006) The validity and reliability of a novel activity monitor as a measure of walking. Br J Sports Med 40:779-784

26. Healy GN, Winkler EA, Owen N, Anuradha S, Dunstan DW (2015) Replacing sitting time with standing or stepping: associations with cardio-metabolic risk biomarkers. Eur Heart J 36:2643-2649

27. Torjesen PA, Birkeland KI, Anderssen SA, Hjermann I, Holme I, Urdal P (1997) Lifestyle changes may reverse development of the insulin resistance syndrome. Oslo Diet Exerc Study: Randomized Trial. Diabetes Care 20:26-31

28. Wajchenberg BL (2010) Clinical approaches to preserve beta-cell function in diabetes. Adv Exp Med Biol 654:515-535

29. Lira VA, Benton CR, Yan Z, Bonen A (2010) PGC-1alpha regulation by exercise training and its influences on muscle function and insulin sensitivity. Am J Phys Endocrinol Metab 299:E145-E161

30. Benton CR, Nickerson JG, Lally J et al (2008) Modest PGC-1alpha overexpression in muscle in vivo is sufficient to increase insulin sensitivity and palmitate oxidation in subsarcolemmal, not intermyofibrillar, mitochondria. J Biol Chem 283:4228-4240

31. Leloup C, Tourrel-Cuzin C, Magnan C et al (2009) Mitochondrial reactive oxygen species are obligatory signals for glucose-induced insulin secretion. Diabetes 58:673-681

32. Robertson RP (2009) Beta-cell deterioration during diabetes: what's in the gun? Trends Endocrinol Metab 20:388-393

33. Robertson RP, Harmon J, Tran PO, Tanaka Y, Takahashi H (2003) Glucose toxicity in beta-cells: type 2 diabetes, good radicals gone bad, and the glutathione connection. Diabetes 52:581-587

34. Malhotra JD, Kaufman RJ (2007) Endoplasmic reticulum stress and oxidative stress: a vicious cycle or a double-edged sword? Antioxid Redox Signal 9:2277-2293

35. Tjonna AE, Lee SJ, Rognmo O et al (2008) Aerobic interval training versus continuous moderate exercise as a treatment for the metabolic syndrome: a pilot study. Circulation 118:346-354

36. Hood MS, Little JP, Tarnopolsky MA, Myslik F, Gibala MJ (2011) Low-volume interval training improves muscle oxidative capacity in sedentary adults. Med Sci Sports Exerc 43:1849-1856

37. Burgomaster KA, Howarth KR, Phillips SM et al (2008) Similar metabolic adaptations during exercise after low volume sprint interval and traditional endurance training in humans. J Physiol 586: $151-160$

38. Little JP, Gillen JB, Percival ME et al (2011) Low-volume highintensity interval training reduces hyperglycemia and increases muscle mitochondrial capacity in patients with type 2 diabetes. J Appl Physiol 111:1554-1560

39. Baldelli S, Aquilano K, Ciriolo MR (2014) PGC-1alpha buffers ROS-mediated removal of mitochondria during myogenesis. Cell Death Dis 5:e1515

40. Ramos JS, Dalleck LC, Tjonna AE, Beetham KS, Coombes JS (2015) The impact of high-intensity interval training versus moderate-intensity continuous training on vascular function: a systematic review and meta-analysis. Sports Med (Auckland, NZ) 45: 679-692

41. Madsen SM, Thorup AC, Overgaard K, Bjerre M, Jeppesen PB (2015) Functional and structural vascular adaptations following 8 weeks of low volume high intensity interval training in lower leg of type 2 diabetes patients and individuals at high risk of metabolic syndrome. Arch Physiol Biochem 121:178-186

42. Madsen SM, Thorup AC, Bjerre M, Jeppesen PB (2015) Does 8 weeks of strenuous bicycle exercise improve diabetes-related inflammatory cytokines and free fatty acids in type 2 diabetes patients 
and individuals at high-risk of metabolic syndrome? Arch Physiol Biochem 121:129-138

43. Malin SK, Solomon TP, Blaszczak A, Finnegan S, Filion J, Kirwan JP (2013) Pancreatic beta-cell function increases in a linear doseresponse manner following exercise training in adults with prediabetes. Am J Phys Endocrinol Metab 305:E1248-E1254

44. Koranyi LI, Bourey RE, Slentz CA, Holloszy JO, Permutt MA (1991) Coordinate reduction of rat pancreatic islet glucokinase and proinsulin mRNA by exercise training. Diabetes 40:401-404

45. Slentz CA, Tanner CJ, Bateman LA et al (2009) Effects of exercise training intensity on pancreatic beta-cell function. Diabetes Care 32:1807-1811

46. Dube JJ, Allison KF, Rousson V, Goodpaster BH, Amati F (2012) Exercise dose and insulin sensitivity: relevance for diabetes prevention. Med Sci Sports Exerc 44:793-799

47. Church TS, Blair SN, Cocreham S et al (2010) Effects of aerobic and resistance training on hemoglobin A1c levels in patients with type 2 diabetes: a randomized controlled trial. Jama 304:2253-2262

48. Madsen SM, Thorup AC, Overgaard K, Jeppesen PB (2015) High intensity interval training improves glycaemic control and pancreatic beta cell function of type 2 diabetes patients. PLoS One 10, e0133286

49. Mitranun W, Deerochanawong C, Tanaka H, Suksom D (2014) Continuous vs interval training on glycemic control and macroand microvascular reactivity in type 2 diabetic patients. Scand $\mathrm{J}$ Med Sci Sports 24:e69-e76
50. Malin SK, Kirwan JP (2012) Fasting hyperglycaemia blunts the reversal of impaired glucose tolerance after exercise training in obese older adults. Diabetes Obes Metab 14:835-841

51. Malin SK, Braun B (2016) Impact of Metformin on exerciseinduced metabolic adaptations to lower type 2 diabetes risk. Exerc Sport Sci Rev 44:4-11

52. Malin SK, Gerber R, Chipkin SR, Braun B (2012) Independent and combined effects of exercise training and metformin on insulin sensitivity in individuals with prediabetes. Diabetes Care 35:131-136

53. Karstoft K, Winding K, Knudsen SH et al (2014) Mechanisms behind the superior effects of interval vs continuous training on glycaemic control in individuals with type 2 diabetes: a randomised controlled trial. Diabetologia 57:2081-2093

54. Krotkiewski M, Lonnroth P, Mandroukas K et al (1985) The effects of physical training on insulin secretion and effectiveness and on glucose metabolism in obesity and type 2 (non-insulin-dependent) diabetes mellitus. Diabetologia 28:881-890

55. Solomon TP, Haus JM, Kelly KR, Rocco M, Kashyap SR, Kirwan JP (2010) Improved pancreatic beta-cell function in type 2 diabetic patients after lifestyle-induced weight loss is related to glucosedependent insulinotropic polypeptide. Diabetes Care 33:1561-1566

56. Dela F, von Linstow ME, Mikines KJ, Galbo H (2004) Physical training may enhance beta-cell function in type 2 diabetes. Am J Phys Endocrinol Metab 287:E1024-E1031 